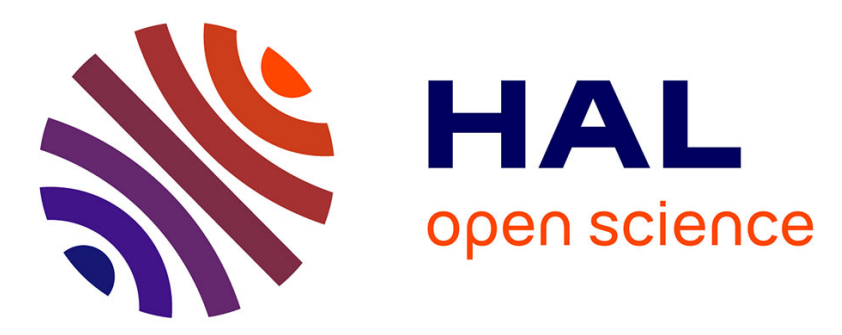

\title{
Slow motion of a sphere towards a plane through confined non-Newtonian fluid
}

Antoine Despeyroux, Abdelhak Ambari

\section{To cite this version:}

Antoine Despeyroux, Abdelhak Ambari. Slow motion of a sphere towards a plane through confined non-Newtonian fluid. Journal of Non-Newtonian Fluid Mechanics, 2012, 167-168, pp.38-45. 10.1016/j.jnnfm.2011.10.001 . hal-01061928

\section{HAL Id: hal-01061928 \\ https://hal.science/hal-01061928}

Submitted on 8 Sep 2014

HAL is a multi-disciplinary open access archive for the deposit and dissemination of scientific research documents, whether they are published or not. The documents may come from teaching and research institutions in France or abroad, or from public or private research centers.
L'archive ouverte pluridisciplinaire HAL, est destinée au dépôt et à la diffusion de documents scientifiques de niveau recherche, publiés ou non, émanant des établissements d'enseignement et de recherche français ou étrangers, des laboratoires publics ou privés. 


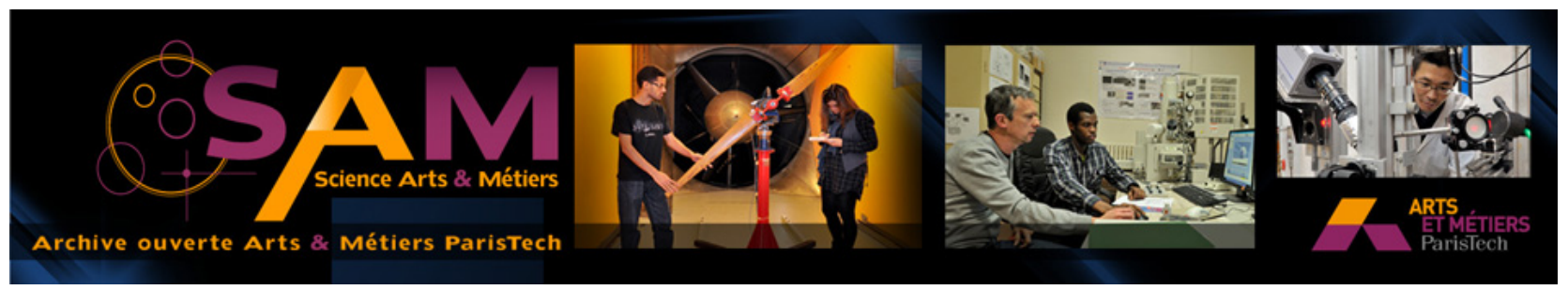

Science Arts \& Métiers (SAM)

is an open access repository that collects the work of Arts et Métiers ParisTech researchers and makes it freely available over the web where possible.

This is an author-deposited version published in: http://sam.ensam.eu

Handle ID: .http://hdl.handle.net/10985/8481

\section{To cite this version :}

Antoine DESPEYROUX, Abdelhak AMBARI - Slow motion of a sphere towards a plane through confined non-Newtonian fluid - Journal of Non-Newtonian Fluid Mechanics - Vol. 167-168, p.38$45-2012$ 


\title{
Slow motion of a sphere towards a plane through confined non-Newtonian fluid
}

\author{
Antoine Despeyroux, Abdelhak Ambari* \\ Arts et Métiers ParisTech, 2 bd du Ronceray, B.P. 3525, 49035 Angers, France
}

\begin{abstract}
A B S T R A C T
The time needed for the contact of two spheres or a sphere with a rigid plane is mainly controlled by the hydrodynamic drainage of the film located in the gap as long as its thickness is out of range of the Van der Waals interactions. In fact, this time controls the dynamics of aggregation of concentrated dispersions. This fundamental problem has an exact solution in Newtonian fluid which has been used to confirm the validity of the numerical dynamic mesh method employed in this geometrically unsteady problem. Following this validation, we applied it to calculate the correction factor of the drag undergone by a sphere approaching a plane, at constant Reynolds number, in a cylindrical tube filled with a non-Newtonian fluid having negligible viscoelastic component and roughly behaving as a power-law fluid. After a justification for using this useful model, we studied the influence of the lateral confinement on the frontal correction factor of the drag. In the lubrication limit, we recall the asymptotic solution of Rodin to this problem in lateral unbounded power law fluid. The comparison of both asymptotical and numerical results confirms their validity. The results obtained in this study may find an application to Dynamic Surface Force Apparatus for nanorheology.
\end{abstract}

\section{Introduction}

The dynamics of non dilute suspensions of particles depends greatly on the hydrodynamic interactions between particles or particles and walls. Among these interactions, those which take place in a frontal way control the time of their contact or separation. This time plays a main role in the aggregation and the formation of the plug flow during the transportation of these suspensions as stressed in particular by de Gennes [1]. This problem is of fundamental interest in many industrial situations (e.g. in filtration, fluidization, sedimentation, etc.). Then, this study deals with the calculation of the Stokes type law correction factor, for the hydrodynamic resistance of a sphere of radius $a$ moving at steady velocity $U$, towards or away from a plane in Newtonian or nonNewtonian fluids. Note that recent applications of Dynamic Surface Force Apparatus (DSFA) in nanorheology $[2,3]$ need the knowledge of this kind of hydrodynamic interaction.

We will focus on the case where the moving velocity $U$ is maintained constant at fixed low Reynolds number in a non-Newtonian fluid of a given apparent viscosity which is dependent only on the second invariant of the strain rate tensor. As the problem is geometrically unsteady, due to the linear variation of the approach distance $d=\varepsilon a$ of the sphere to the plane in time, we assume that a quasi-steady solution applies and the history force and the added mass force are irrelevant in this case.

\footnotetext{
* Corresponding author. Tel.: +33 241207362; fax: +33 241207362

E-mail address: ambari@ensam.eu (A. Ambari).
}

$\varepsilon \operatorname{Re} \ll 1$ constitutes the condition of quasi-steady flow, as expressed by Cox and Brenner [4], where $\operatorname{Re}=2 a U / v$ and $v$ is the kinematic viscosity. This condition can be found by assuming that the vorticity in the gap induced by the relative motion of the sphere with respect to the plane, must be established in a characteristic time $(\varepsilon a)^{2} / v$ lower than the unsteady convective one $\varepsilon a / U$. The results given in this paper may be applicable to the sedimentation of a particle in the case where the added mass and the history forces, as the inertia, are negligible. Using bipolar coordinates which were first used by Stimson and Jeffrey [5], in the lateral unbounded Newtonian fluid, Brenner [6] and Maude [7] calculated analytically the correction factor $\delta(\varepsilon)$ defined by:

$$
\delta(\varepsilon)=\frac{F(\varepsilon)}{6 \pi \mu a U}
$$

For the small gap $\varepsilon a$ between the plane and the sphere, the asymptotic expansion of their formula is given by Cox and Brenner [4]: $\delta(\varepsilon)=\varepsilon^{-1}\left(1-\frac{1}{5} \varepsilon \ln \left(\frac{1}{\varepsilon}\right)+0.9712 \varepsilon\right)$ which is valid for $\varepsilon \leqslant 0.6$. The first term of this asymptotic expansion is the well-known Taylor law $\delta(\varepsilon)=\varepsilon^{-1}$ which is valid only for $\varepsilon \leqslant 0.4$. Experimentally, the Brenner formula has been successfully verified by Ambari et al. [8].

As the sedimentation often occurs in a confined situation due to the presence of lateral walls or other particles, we will quantify the influence of the lateral hydrodynamic interaction relative to the frontal correction factor. Still in the Newtonian case, the effect of the lateral confinement induced by the wall of an infinitely long circular cylinder has been calculated numerically and experimentally by Ambari et al. [9] and Ben Richou et al. [10]. Apart from 
the asymptotic calculation given by Rodin [11] in lateral unbounded power-law fluid, for the squeezing motion of two nearly-touching rigid spheres, there are other similar asymptotic solutions for non-Newtonian fluids [12] to the same problem. Otherwise, as far as we know, there are no numerical results given in non-Newtonian fluid concerning the calculation of the drag undergone by a sphere translating at constant velocity towards a plane. To study the first non-Newtonian effect, we focused on the shear thinning or shear thickening behavior of these fluids. Then we give a numerical solution to this problem in power-law fluids whose constitutive equation can be described as follows:

$\sigma_{i j}=-p \delta_{i j}+2 m\left|2 d_{k l} d_{k l}\right|^{(n-1) / 2} d_{i j}$

where $\sigma_{i j}$ are the Cauchy stress tensor components, $d_{i j}=\frac{1}{2}\left(U_{i, j}+U_{j, i}\right)$ the rate of deformation tensor components, $p$ the pressure, $m$ and $n$ are respectively the consistency coefficient $\left(\mathrm{Pas}^{n}\right)$ and the powerlaw index of the fluid. Concerning the drag undergone by a particle moving towards a plane, this is mainly due to the fluid drainage in the gap in the lubrication regime. Then, the non-Newtonian behavior of the fluid which takes place in this drainage flow may be, in first approximation, modeled by a power-law fluid because of the weakness of the elongational gradient, which is able to arouse the viscoelasticity of the fluid, due to the very low velocity of the particle as made by Sherwood [13]. Moreover, experimental results on particle-wall collision in polymeric liquids given by Ardekani et al. [14] also justify the choice of the power-law model for this problem. Note that the pressure induced by the drainage flow is the main contributory factor to the drag, and the shear velocity gradient is limited by its maximum value achieved near the symmetry axis. When the fluid has a shear thinning behavior, a possible first Newtonian plateau corresponding to low shear velocity gradient, situated in the extreme vicinity and far from the axis of the sphere, has very little contribution in the calculation of the force. So the Ostwald model can constitute a good approximation. For the shear thickening fluids, a similar analysis is applicable. The use of the Ostwald handy model involving only one control parameter, which is the fluidity index $n$, contrary to that of a more realistic model such as Carreau-Yasuda introducing four control parameters [15], enables us to clearly and physically show the influence of the shear thinning and the shear thickening behavior on the drag undergone by this particle.

\section{Formulation and numerical methodology}

To calculate the frontal correction factor $\delta(n, k, \varepsilon)$ in lateral confined medium defined by $k$, we consider the flow induced by a spherical particle, of radius $a$, translating axially towards the bottom at constant velocity $\boldsymbol{u}_{z}=-U \boldsymbol{e}_{z}$ inside a cylindrical tube of radius $b$, as shown schematically in Fig. 1. This container is filled with a Newtonian or non-Newtonian fluid. To make easier the numerical calculations, we consider an equivalent configuration where the sphere is set and the container is moving at the velocity $+U \boldsymbol{e}_{z}$. The flow is governed by the momentum and mass conservation equations under isothermal conditions, i.e.

$\rho\left(\partial_{t} \boldsymbol{U}+(\boldsymbol{U} \cdot \boldsymbol{\nabla}) \boldsymbol{U}\right)=-\nabla p+\nabla \cdot \underline{\underline{\tau}}$

$\boldsymbol{\nabla} \cdot \boldsymbol{U}=0$

where $\rho$ is the fluid density, $p$ the pressure and $\tau=\left(\sigma_{i j}+p \delta_{i j}\right)$ the extra-stress tensor for the power-law fluid (see Eq. (2)). The velocity boundary and initial conditions are defined by: (i) on all the walls of the cylinder: $\boldsymbol{U}=+U \boldsymbol{e}_{z}$; (ii) on the sphere: $\boldsymbol{U}=0$; (iii) for $t \leqslant 0$ the fluid is at rest: $\boldsymbol{U}=0$.

At low and fixed generalized Reynolds numbers $\operatorname{Re}_{n}=$ $\rho U^{2-n}(2 a)^{n} / m$, the frontal and lateral correction factor $\delta(n, k, \varepsilon)$, due to the presence of the lateral and frontal walls, of the drag

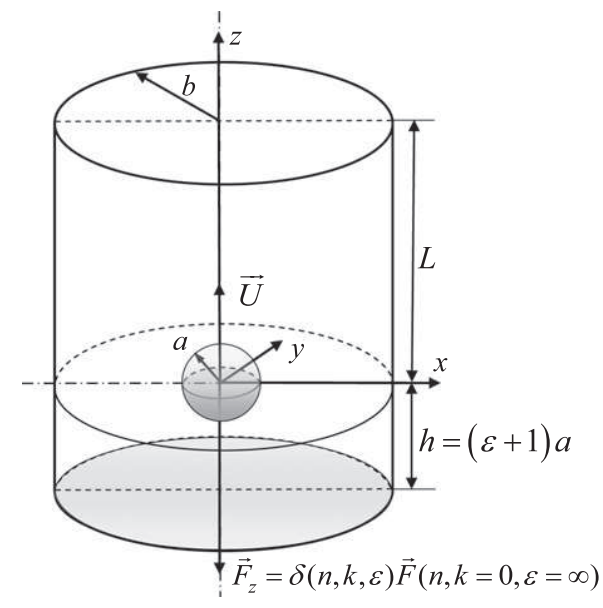

Fig. 1. Geometrical and dynamical parameters definition of the sphere moving towards the bottom in the axis of a cylindrical tube.

undergone by a sphere translating towards the plane bottom in the axis of the cylinder is calculated through the following expression obtained by dimensional analysis:

$\delta(n, k, \varepsilon)=\frac{F(n, k, \varepsilon)}{6 \pi m\left(\frac{U}{2 a}\right)^{n-1} a U}$

where $k=a / b$ is the lateral confinement factor and $\varepsilon=d / a$ is the frontal. In unbounded fluids, $\delta(n, k=0, \varepsilon=\infty)$ has been calculated by us numerically [16]. In this case, the correct values of this coefficient are given with an average relative error of less than $1 \%$ for $0 \leqslant n \leqslant 1.8$ by the polynomial interpolation formula:

$$
\begin{aligned}
\delta(n, k=0, \varepsilon=\infty)= & 1.191+1.978 n-4.916 n^{2}+7.333 n^{3} \\
& -8.717 n^{4}+5.743 n^{5}-1.838 n^{6}+0.228 n^{7}
\end{aligned}
$$

The numerical solution of this geometrically unsteady problem is obtained using the dynamic mesh method in the finite volume CFD FLUENT code where the SIMPLE algorithm was employed with a second order scheme. In the integral form, the continuity and momentum equations are solved through the dynamic mesh technique in FLUENT [17, p. 36], on a control volume $V$, involving a moving boundary $\partial V$ :

$\frac{\mathrm{d}}{\mathrm{d} t} \int_{V} \rho \boldsymbol{U} \mathrm{d} V+\int_{\partial V} \rho \boldsymbol{U}\left(\boldsymbol{U}-\boldsymbol{U}_{g}\right) \cdot \mathrm{d} \boldsymbol{S}=-\int_{\partial V} p \underline{\underline{I}} \cdot \mathrm{d} \boldsymbol{S}+\int_{\partial V} \underline{\underline{\tau}} \cdot \mathrm{d} \boldsymbol{S}$

$\int_{\partial V} \rho \boldsymbol{U} \cdot \mathrm{d} \boldsymbol{S}=0$

where $\rho$ is the fluid density, $\boldsymbol{U}$ the fluid velocity, $\boldsymbol{U}_{g}$ the moving mesh velocity, $p$ the pressure, $\underline{\underline{I}}$ the identity matrix, and $\underline{\underline{\tau}}$ the extra-stress tensor. The time-derivative term is computed using a first-order backward formula:

$\frac{\mathrm{d}}{\mathrm{d} t} \int_{V} \rho \boldsymbol{U} \mathrm{d} V=\frac{(\rho \boldsymbol{U} V)^{l+1}-(\rho \boldsymbol{U} V)^{l}}{\Delta t}$

where $l$ and $l+1$ denote, respectively the quantity at the current and following step. The volume $V^{l+1}$ is obtained through the relation:

$V^{l+1}=V^{l}+\frac{\mathrm{d} V}{\mathrm{~d} t} \Delta t$

where

$\frac{\mathrm{d} V}{\mathrm{~d} t}=\int_{\partial V} \boldsymbol{U}_{\boldsymbol{g}} \cdot \mathrm{d} \boldsymbol{S}=\sum_{j}^{N_{f}} \boldsymbol{U}_{g, j} \cdot \boldsymbol{S}_{j}$ 
where $N_{f}$ is the number of faces of the control volume $V, \boldsymbol{S}_{j}$ is the $j$ face area vector, and $\boldsymbol{U}_{g . j} \cdot \boldsymbol{S}_{j}=\frac{\delta V_{j}}{\Delta t}$ with $\delta V_{j}$ the volume covered by the face $j$ over the time step $\Delta t$. These calculations are carried out on a structured mesh and a non structured one only in the vicinity of the stagnation point of the sphere to ensure a homogeneous fixed mesh size during the deformation of the mesh. For each time step, this dynamic mesh technique involves the rigid motion of the top and bottom of the container, respectively, away from and towards the boundary of the sphere. The mesh is then adjusted according to the new position of the moving boundaries. In the rectangular mesh domain near the bottom, the dynamic layering removes layers of cells adjacent to the moving boundary (bottom), based on the height of the layer adjacent to the moving surface. For this reason, the cells are split (top) or merged (bottom) with the layer of cells next to them when their layer attains a critical height. The minimum thickness of the non structured mesh zone at the stagnation point of the sphere $\left(\varepsilon=10^{-3}\right)$ corresponds to the minimum gap which can be reached through this procedure. Let us recall that a hundred iterations for each time step is used. The distance from the sphere to the top of the tube $L$ is given equal to $60 a$ to avoid the influence of the top of the tube on the drag. For this computation, we used a sixteen core cluster. The computation at each step $l$ is supposed to be converged when the following criterion is verified: $\left|1-\delta^{l}(n, k, \varepsilon)\right| \delta^{l+1}(n, k, \varepsilon) \mid<10^{-6}$. Furthermore, it is important to note that as far as we are concerned, in this particular flow, by a bounded shear stress $\tau_{r z}$ corresponding to a bounded shear rate $0 \leqslant \dot{\gamma}_{r z} \leqslant \dot{\gamma}_{r z \max }$, there is no singularity in the shear stress, even if the apparent viscosity described by this model mathematically diverges at a zero shear rate. Finally, note that the domains in which the velocity gradient (then the shear stress) is negligible do not introduce a significant contribution to the calculation of the drag. In this condition, we proceeded to a verification of the effect of a truncated Ostwald model at low and high velocity gradients as it would be obtained by the Carreau-Yasuda model [15]. Indeed, according to most of the rheological experimental results [15] showing Newtonian plateaus, we imposed a variation of the apparent viscosity over three decades truncated at low and high shear velocity gradients. This variation, centered around the value corresponding to the mean velocity gradient achieved at $\theta=\pi / 2$, has been imposed. As we will see in Fig. $5 \mathrm{a}$ in Section 4.2, all the results corresponding to this truncated Ostwald model remain the same as those obtained using the complete Ostwald model.

In the lubrication regime, we proceeded to an asymptotic approach to check the validity of the results obtained by the dynamic mesh numerical method employed in this study.

\section{Asymptotic results}

In the limit of the lubrication regime $\varepsilon \ll 1$ and for very low Reynolds numbers, when the sphere moves towards the plane in unbounded lateral medium (see Fig. 2), the drag force is controlled principally by the drainage process of the liquid film located in the minimum gap remaining between the sphere and the plane. Otherwise, in this limit, Rodin [11] has given an asymptotic solution for the squeezing motion of two nearly-touching rigid spheres $\left(S_{1}\right.$ of radius $a$ and $S_{2}$ of radius $\beta a$ ) in a power-law fluid. To solve this problem, he used the axisymmetric Stokes stream function so the asymptotic problem is analyzed in non dimensional stretched coordinates. He calculated the asymptotic solution for the pressure for different values of the $\alpha=(1+\beta) / 2 \beta$ parameter, and deduced the asymptotic solution for the drag undergone by each sphere, by integrating the pressure transmitted by a horizontal circle of radius $a$ centered at the origin. Concerning our configuration of the sphere settling towards a plane, we took $\beta \rightarrow \infty$ then $\alpha=1 / 2$. When we replace this $\alpha$ value in his solution for the pressure we obtain this radial distribution of the pressure: (a)

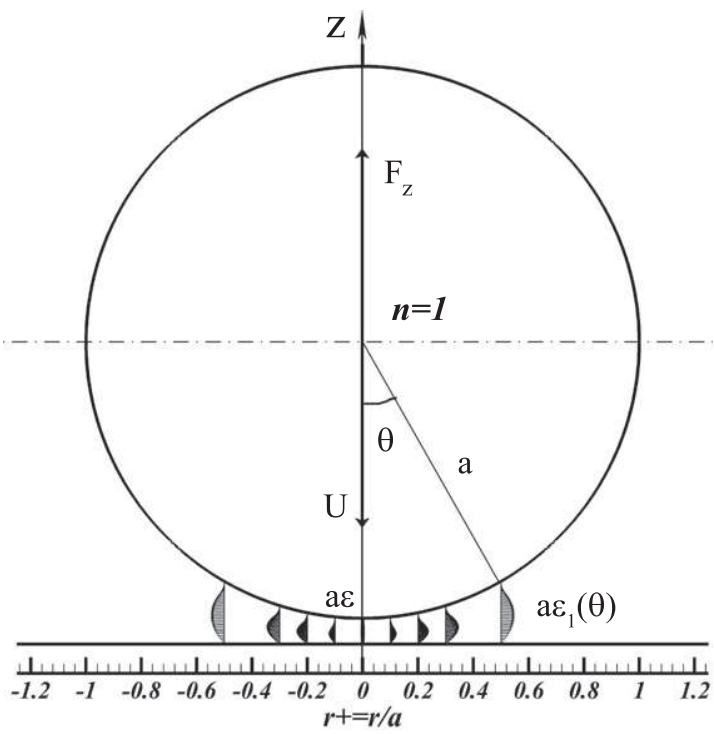

(b)

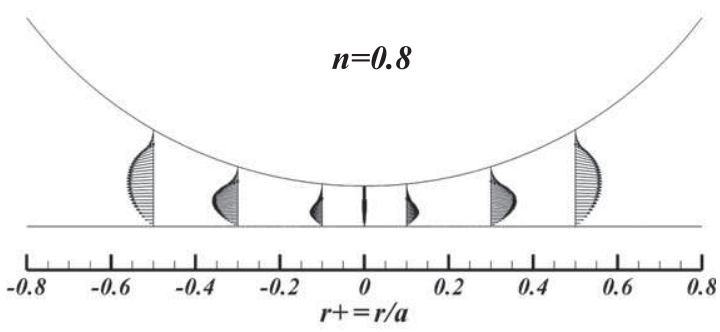

(c)

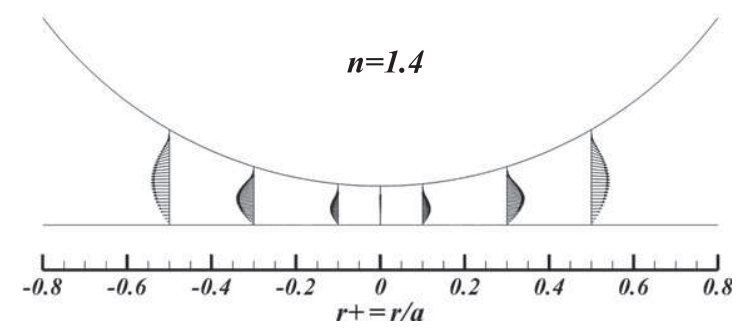

Fig. 2. Numerical velocity field in the gap between the sphere and the plane when the sphere is in the vicinity of the bottom $\left(\varepsilon=10^{-2}, k=10^{-2}, \operatorname{Re}_{n}=10^{-3}\right):(\mathrm{a}) n=1$, (b) $n=0.8$, and (c) $n=1.4$.

$$
\begin{aligned}
\frac{p\left(r_{+}\right)-p_{\infty}}{m\left(\frac{U}{2 a}\right)^{n}}= & \left(\frac{2 n+1}{n}\right)^{n}\left(\frac{2^{2+3 n}}{1+3 n}\right)\left(r_{+}\right)^{-(1+3 n)} \\
& \times{ }_{2} F_{1}\left(1+2 n, \frac{1}{2}(1+3 n) ; \frac{3}{2}(1+n) ;-2 \frac{\varepsilon}{r_{+}^{2}}\right)
\end{aligned}
$$

where $p_{\infty}$ is the pressure far from the gap, $r_{+}=r / a$ is the normalized radial distance from the stagnation point and ${ }_{2} F_{1}$ is the Gaussian hypergeometric function. For $n=1$ corresponding to the Newtonian fluid, this expression reduces to:

$$
\frac{p\left(r_{+}\right)-p_{\infty}}{\mu\left(\frac{U}{2 a}\right)}=\frac{6}{\left[\varepsilon+\frac{1}{2} r_{+}^{2}\right]^{2}}
$$

which is the same as the classical lubrication solution [18-20]. In this approach, the correction factor $\delta(n, k=0, \varepsilon)$ can also be obtained for $\alpha=1 / 2$ in the expression of the force given by Rodin [11]:

$$
\begin{aligned}
\delta(n, k & =0, \varepsilon)=\frac{F(n, k=0, \varepsilon)}{F(n, k=0, \varepsilon \rightarrow \infty)} \\
& =\frac{2^{\frac{3 n-1}{2}}}{3}\left(\frac{2 n+1}{n}\right)^{n} \beta\left(\frac{3+n}{2}, \frac{3 n-1}{2}\right) \frac{1}{\varepsilon^{\frac{3 n-1}{2}}}
\end{aligned}
$$




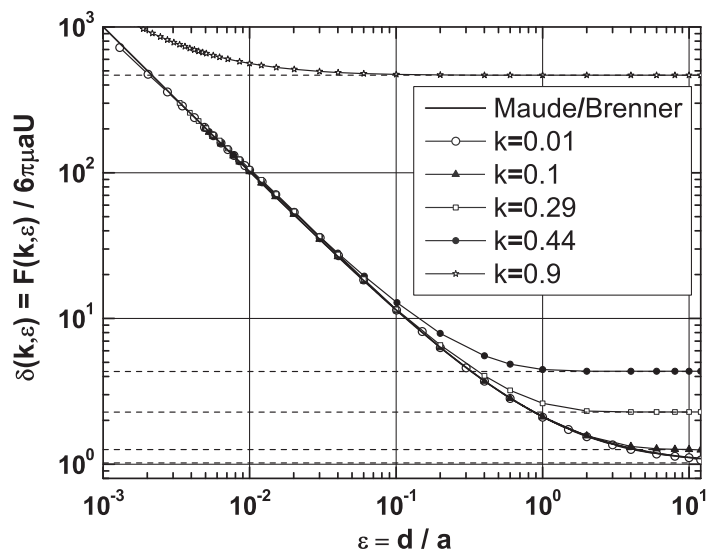

Fig. 3. The effect of lateral confinement on the frontal correction factor of the drag force undergone by a sphere moving axially towards the bottom of a tube filled with a Newtonian fluid. The dashed plateau lines represent $\lambda(k)$.

Table 1

Critical thicknesses of the minimum gap $\varepsilon_{c}$ at which the drag deviates (of $1 \%$ ) from its plateau corresponding to its value achieved far from the bottom of the tube.

\begin{tabular}{llllll}
$k$ & 0.01 & 0.1 & 0.29 & 0.44 & 0.9 \\
$\varepsilon_{c}$ & 62 & 6.7 & 2.2 & 1.28 & 0.114 \\
\hline
\end{tabular}

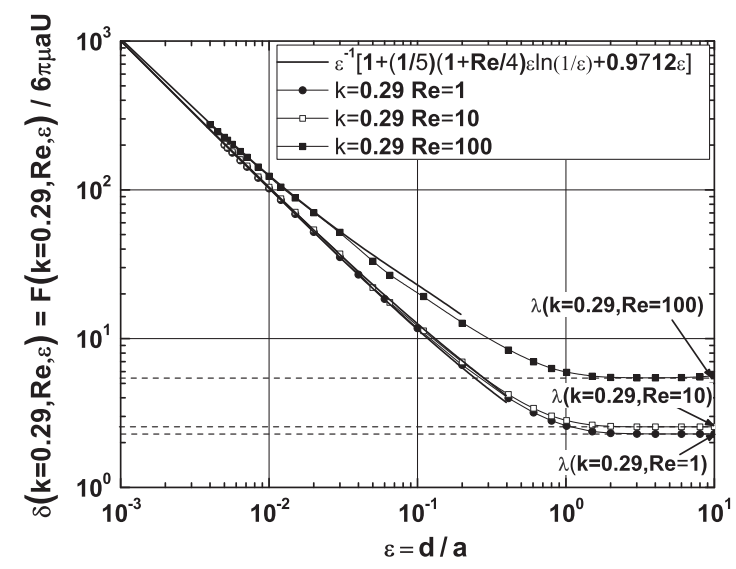

Fig. 4. Influence of the inertia on the drag undergone by a sphere moving towards a plane $(k=0.29)$, and comparison with Cox and Brenner's asymptotic relation (Eq. (17)) for $\operatorname{Re}=1,10$ and 100 .

This formula which is valid mathematically only for $n>1 / 3$ in the Newtonian case, reduces to the classical Taylor solution:

$\delta(n=1, k=0, \varepsilon)=\frac{1}{\varepsilon}$

Otherwise we propose in Appendix A a simple asymptotic solution corresponding to the sphere moving towards a plane in the limit of the lubrication regime and avoiding the use of the stream function. This calculation gives a similar asymptotic solution to that obtained from Rodin's formula corresponding to $\beta=\infty$.

\section{Results and discussion}

First, we give a comparison of the numerical and asymptotical results obtained for Newtonian fluid with the aim of giving a mutual validation of the numerical method and asymptotical approach, followed by the results concerning the relative effect between both lateral and frontal confinements. In the second step, (a)

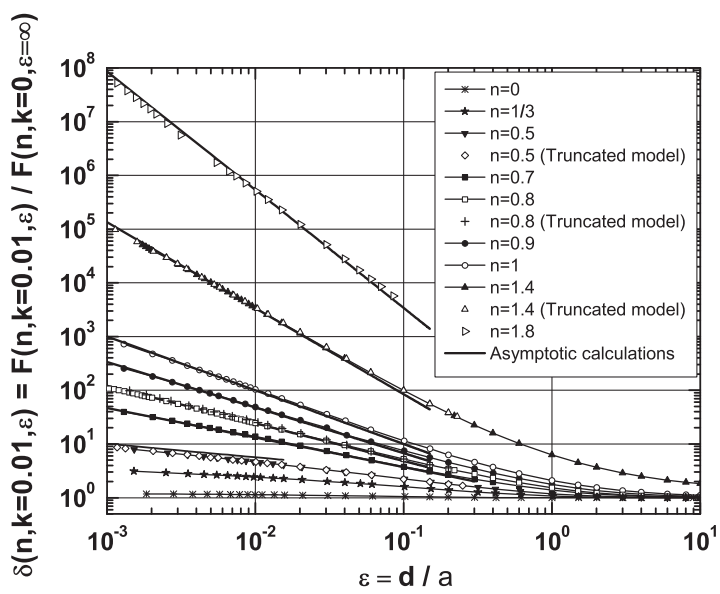

(b)

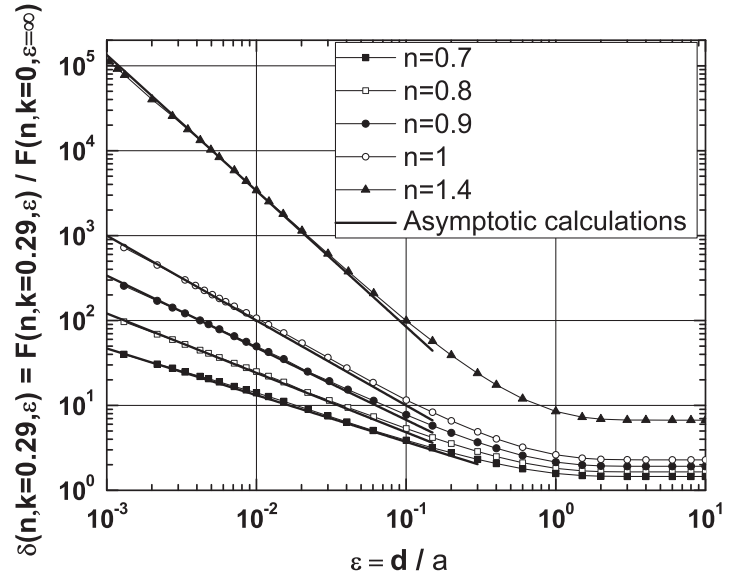

(c)

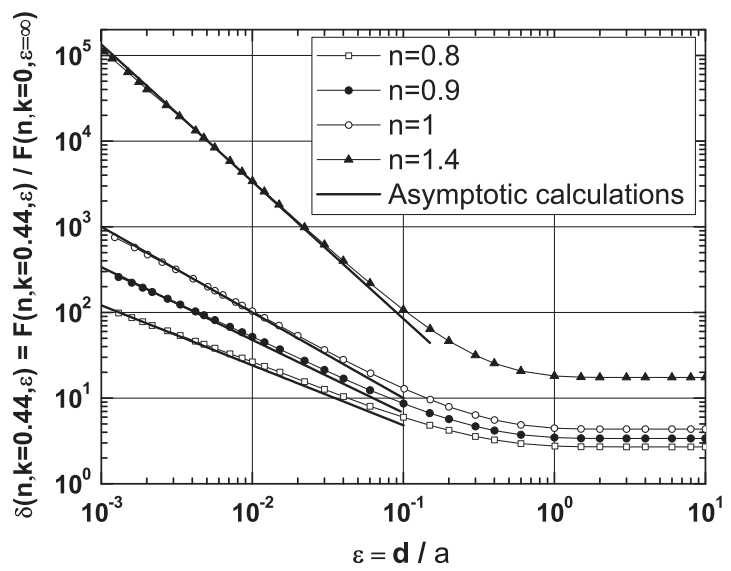

Fig. 5. Influence of the fluidity index on the drag undergone by a sphere approaching axially the plane bottom of a tube filled with a power-law fluid, for different confinements and comparison between the numeric (scatter) and asymptotic (solid line) results: (a) $k=0.01$, (b) $k=0.29$, and (c) $k=0.44$.

we give the non-Newtonian correction factor numerically and asymptotically in the case of the power-law fluid for different indexes of fluidity and different confinements.

\subsection{Newtonian fluid}

As in most experiments, the sphere has to move towards a plane in confined situation according to the sketch Fig. 1, the drag force must be corrected from the effect of the lateral confinement, de- 
(a)

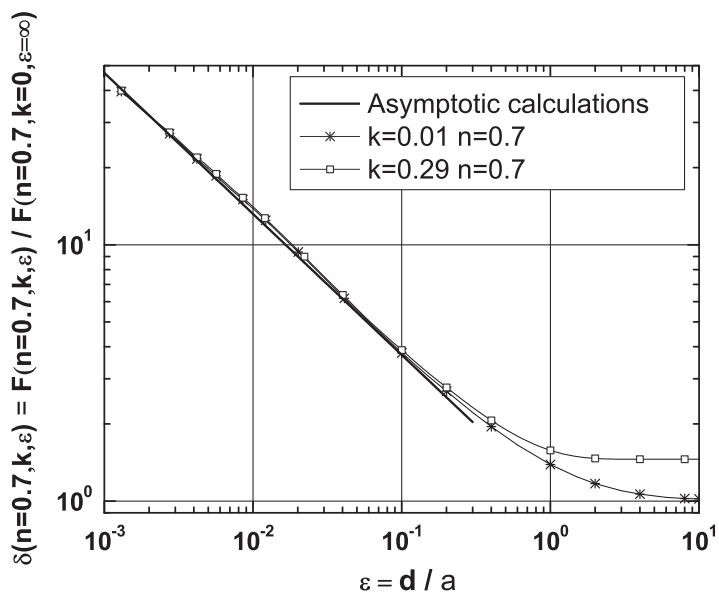

(b)

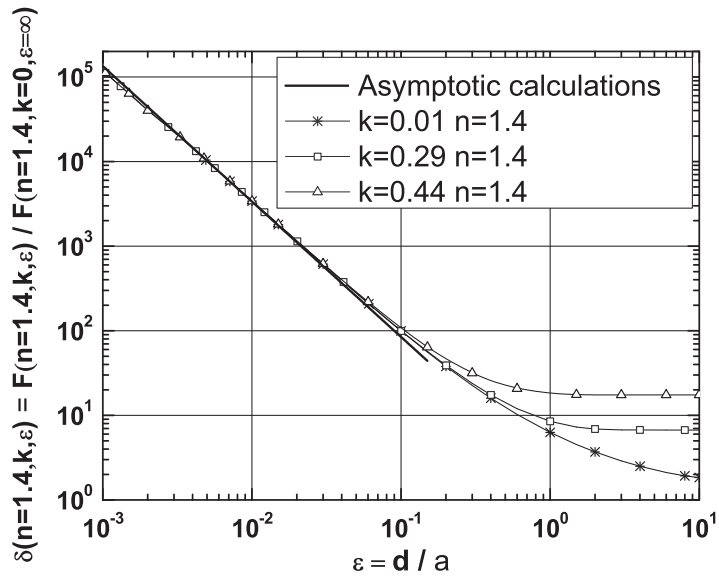

Fig. 6. Absence of the influence of the lateral confinement on the drag undergone by a sphere in a power-law fluid in the lubrication limit, for two fluidity indexes: (a) $n=0.7$, and $(\mathrm{b}) n=1.4$.

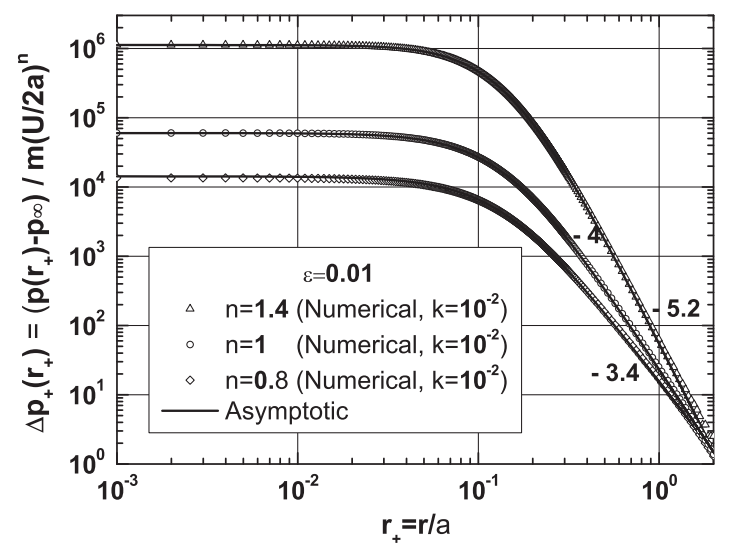

Fig. 7. Comparison of the numerical and the asymptotical pressure distribution in the gap between the sphere and the plane for different fluidity indexes $n=0.8, n=1$ and $n=1.4$, for $\operatorname{Re}=10^{-3}, \varepsilon=10^{-2}$ and $k=10^{-2}$. (a)

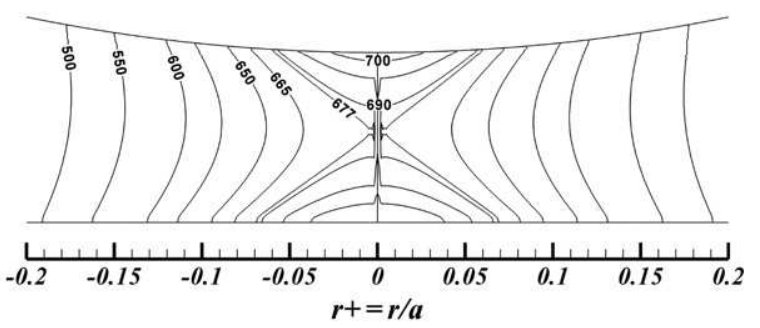

(b)

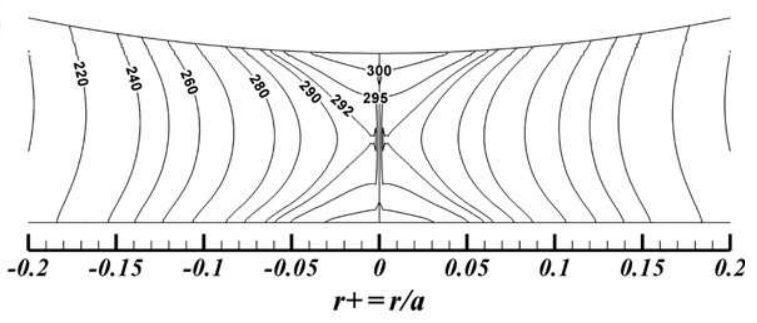

(c)

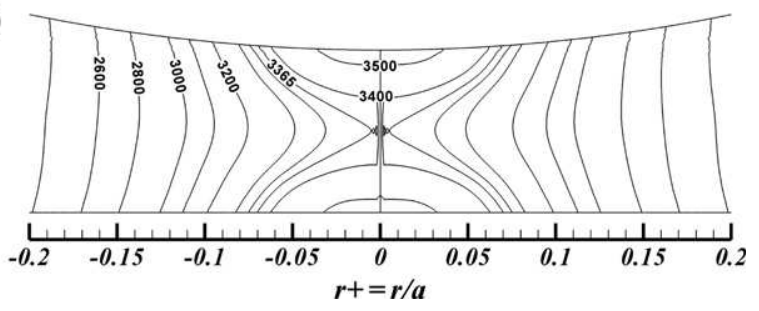

Fig. 8. Bulk normalized pressure distribution $\left(p\left(r_{+}\right)-p_{\infty}\right) / m(U / 2 a)^{n}$ in the gap between the sphere and the bottom showing the appearance of a saddle point in the axis for $\operatorname{Re}=10^{-3}, \varepsilon=10^{-2}$ and $k=10^{-2}$ : (a) $n=1$, (b) $n=0.8$, and (c) $n=1.4$.

fined by the ratio $k=a / b$, and the frontal one defined by the gap $\varepsilon=d / a$. The numerical results obtained for different lateral confinements at $\operatorname{Re}=10^{-3}$, for $0.01 \leqslant k \leqslant 0.9$ given in Fig. 3, show clearly that when the sphere moves towards the bottom of the container, the effect of the frontal correction factor takes place further from the plane, all the more so that $k$ is low. For $k=10^{-2}$, the numerical results we obtained are in good agreement with the exact analytical solution from Maude [7] and Brenner [6]. For this lateral confinement $\left(k=10^{-2}\right)$, at least at a distance of ten sphere radii to the bottom, the lateral correction factor is negligible $\left(\lambda\left(k=10^{-2}, \varepsilon \rightarrow \infty\right)=F\left(k=10^{-2}\right) /(6 \pi \mu a U)=1.017\right.$ [10] and $\delta(\varepsilon=$ $10)=1.109)$. This successful result confirms the validity of the dynamic mesh numerical method used for this unsteady problem. Moreover, the limit of $\delta(k, \varepsilon)$ (its value in the plateau) when $\varepsilon$ is greater than a critical value $\varepsilon_{c}$ (at which the drag deviates from the plateau depending on the lateral confinement see Table 1) is also in good agreement with the obtained value of the lateral correction factor $\lambda(k, \varepsilon \rightarrow \infty)=F(k) / 6 \pi \mu a U[10]$.

$\delta\left(k, \varepsilon>\varepsilon_{c}\right) \rightarrow \lambda(k)$

However, for all confinements, when the sphere is approaching the plane in the lubrication limit, the asymptotic behavior of $\delta(k, \varepsilon)$ is the same as in the lateral unbounded medium. This effect is due to the fact that the lateral correction factor becomes negligible in comparison with the frontal one, in the lubrication regime when $\varepsilon \rightarrow 0$. Moreover, in Fig. 7, the radial distribution of the pressure calculated numerically, at low Reynolds number $\left(\operatorname{Re}=10^{-3}\right)$ and

Table 2

Comparison of the exponents $\beta(n)$ of the power-law behavior obtained numerically and those obtained asymptotically.

\begin{tabular}{|c|c|c|c|c|c|c|c|c|c|c|c|c|}
\hline$n$ & 0 & 0.1 & $1 / 3$ & 0.4 & 0.5 & 0.7 & 0.8 & 0.9 & 1 & 1.4 & 1.8 & 2 \\
\hline$g(n)$ (Numeric) & -0.03 & -0.05 & -0.13 & -0.20 & -0.29 & -0.55 & -0.68 & -0.82 & -0.98 & -1.57 & -2.16 & - \\
\hline$g(n)$ (Asymptotic) & - & - & - & -0.10 & -0.25 & -0.55 & -0.70 & -0.85 & -1 & -1.60 & -2.20 & -2.5 \\
\hline
\end{tabular}


low lateral confinement $\left(k=10^{-2}\right)$, is in good agreement with that calculated asymptotically (Eq. (13)). This agreement corroborates the validity of the numerical method. Taking advantage of the accuracy of this method, we give in Fig. 4 the influence of the inertia on the correction factor $\delta(\varepsilon, k)$ and its asymptotic behavior for a given lateral confinement $k=0.29$ and $\operatorname{Re}=1,10$, and 100 . As the correction of the lateral confinement is negligible in the lubrication regime (see above), the comparison of the numerical results and those given by Cox and Brenner [4] shows a good agreement and confirms, for the first time, to our knowledge, the validity of their asymptotic expression:

$\delta(\varepsilon, \operatorname{Re})=\frac{1}{\varepsilon}+\frac{1}{5}\left(1+\frac{\operatorname{Re}}{4}\right) \ln \left(\frac{1}{\varepsilon}\right)+0.9712$

When $\varepsilon \rightarrow 0$, the influence of the Reynolds number becomes negligible, confirming that the inertia in the lubrication regime is mainly controlled by $\varepsilon$ Re, as pointed out above. Otherwise, this situation, where the Reynolds number is fixed, differs fundamentally from the sedimentation of a buoyant sphere, where its settling velocity decreases continuously, and thus the Reynolds number far from the plane plays an important role, through the Stokes number $\mathrm{St}=(1 / 9)\left(\rho_{s} / \rho_{f}\right) \operatorname{Re}$ (where $\rho_{s}$ and $\rho_{f}$ are respectively the densities of the sphere and the fluid), in the transition from the non bouncing to the bouncing regime [21-24].

\subsection{Non-Newtonian fluid}

To justify the use of the power-law fluid model, let us recall that, in this work, as we consider non-Newtonian fluid with a relaxation time lower than the inverse of the maximum of the normal and radial elongational velocity gradient (located in the vicinity of the stagnation point), the effect of a possible viscoelasticity is negligible [25-27]. In this condition, the behavior of the fluid in this almost Poiseuille-type flow (viscometric one) can be modeled in first approximation by a power-law fluid. Then, in Fig. 5a-c, we show the influence of the index of fluidity $n$ on the correction factor $\delta(n, k, \varepsilon)$ for $k=0.01, k=0.29$ and $k=0.44$. For each of these lateral confinements, the frontal correction factor corresponding to the drag undergone by a sphere at a distance $\varepsilon a$ from the plane, normalized by the same force in unbounded power-law medium, is given by the Eqs. (5) and (6). In Fig. 5a-c, we observe for all confinements, a good agreement between the numerical results and those obtained by the asymptotic approach given by the Eqs. (14) and (A.7) corresponding to the lateral unbounded medium for $n>0.5$. In fact, the influence of the lateral confinement in comparison to the frontal one is very weak in the lubrication regime and depends only on the fluidity index of the fluid through the Eqs. (14) and (A.7), as shown in Fig. 6a for the pseudoplastic fluid $(n=0.7)$ and in Fig. $6 \mathrm{~b}$ for the shear thickening fluid $(n=1.4)$.

Otherwise, concerning Fig. 5a-c, we show that, when the sphere moves towards the plane, the more the fluid is shear thinning, the lower the increase in the frontal correction factor is. Thus, the aggregation of particles in suspensions is facilitated in shear thinning fluids, relative to the Newtonian case. Finally, as discussed in Section 2, to confirm the absence of the effect of a truncation at low and high velocity gradients introduced by the appearance of both Newtonian plateaus as in the Carreau-Yasuda model [15], we numerically calculated the same correction factor with an Ostwald truncated model whose apparent viscosity varies over three decades (as encountered in experiments). The good agreement of the results, given in Fig. 5a, obtained with this truncated Ostwald model with those obtained by employing a complete one, justifies its use in this study.

Concerning the radial distribution of the pressure in the gap, we observe a power-law decrease in the pressure with the radial distance, as in the Newtonian case where $\left(p\left(r_{+}\right)-p_{\infty}\right) \propto r_{+}^{-4}$. As with the Newtonian fluid, we compare successfully in Fig. 7 the

(a)

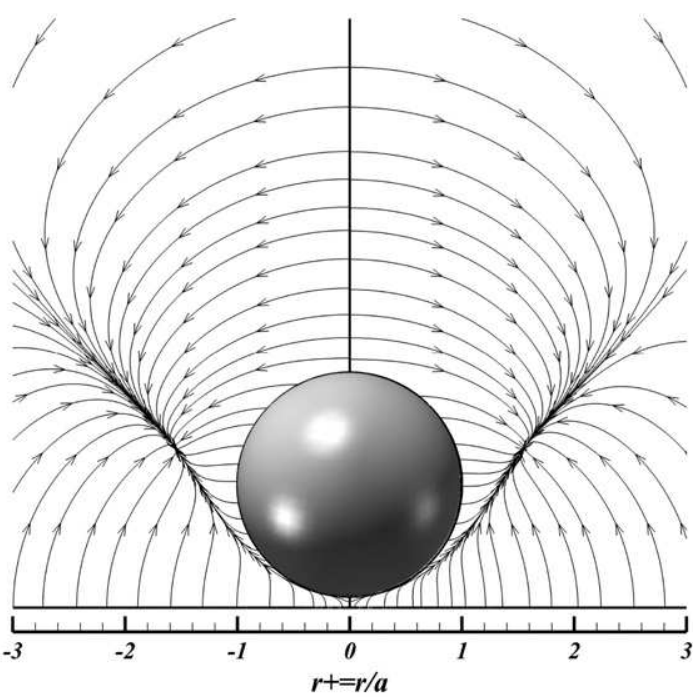

(b)

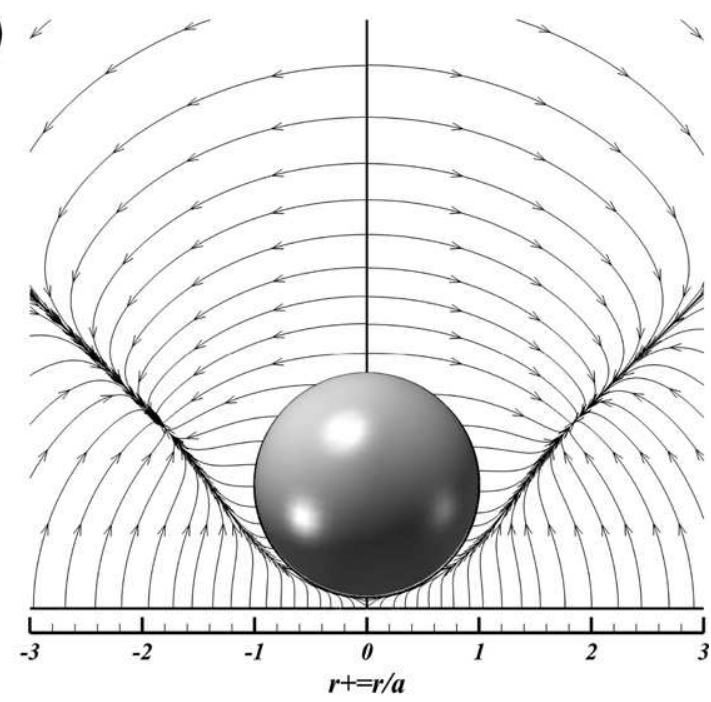

(c)

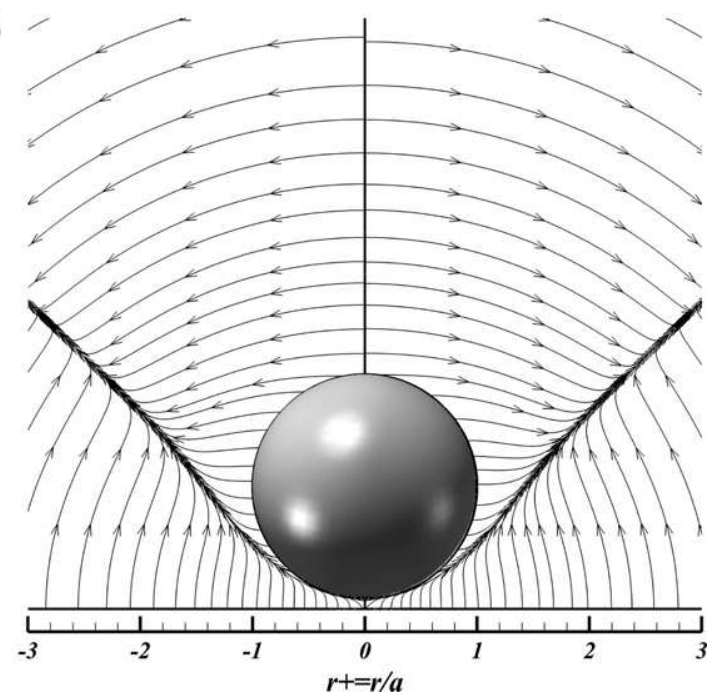

Fig. 9. Instantaneous streamlines of the flow in the frame related to the sphere for different indexes of fluidity $\left(\operatorname{Re}=10^{-3}, \varepsilon=10^{-2}\right.$ and $\left.k=10^{-2}\right)$ : (a) $n=0.8$, (b) $n=1$, and (c) $n=1.4$. 
non-Newtonian numerical $\left(k=10^{-2}\right)$ and asymptotical results (Eqs. (12) and (A.6)) for two indexes of fluidity, corresponding respectively to dilatant $(n=1.4)$ and pseudoplastic $(n=0.8)$ fluids for $\varepsilon=0.01$. In all cases, the numerical results for Newtonian and power-law fluids confirm the validity of the asymptotic relations (12) and (A.6). The same agreement is shown for different $\varepsilon$. Concerning the bulk distribution of the pressure in the gap, Fig. 8 shows that the high pressure domain is located in the lowest gap, for different indexes of fluidity. Then, it is observed that an absolute maximum pressure is attained at the stagnation point of the sphere and at the bottom. The minimum pressure is attained at the saddle point located in the middle of the lowest gap in the symmetry axis. Finally, let us remark that, for the same Reynolds number $\operatorname{Re}_{n}=10^{-3}$, the pressure is reduced in the pseudoplastic fluid and increased in the dilatant one, in comparison to the Newtonian case. Otherwise, concerning the power-law evolution of the drag force in the lubrication regime, we give in Table 2 the exponent of this power-law deduced from the numerical curve given in Fig. 5a and that calculated asymptotically: $g(n)=-(3 n-1) / 2$. The comparison of both results confirms that the asymptotic expressions (14) and (A.7) give accurate results only for $n>0.5$ as discussed above. For $n<0.5$, the agreement no longer remains because the asymptotic calculation needs to be performed at higher asymptotic order. Indeed, for the shear thinning fluid, the part of the sphere which contributes to the drag force is all the more increased as the fluidity index decreases.

To show how the flow field is affected by the fluidity, we give some examples of the instantaneous streamlines of the flow in the frame related to the sphere for different indexes of fluidity $n=0.8, n=1$ and $n=1.4$ in Fig. 9. They show the appearance of the singular instantaneous streamline of the flow and a change of the flow aspect in the dilatant fluid case.

\section{Conclusion}

The power-law which describes the divergence of the drag force undergone by a sphere moving towards a plane, has been numerically and asymptotically studied in Newtonian and non-Newtonian fluids in lateral confined media. The successful confrontation with the exact solution given for a lateral unbounded medium by Maude and Brenner, confirms the validity of the dynamic mesh method employed in this geometrically unsteady problem. Thereby we investigate in the Newtonian case, the effect of inertia and the influence of the lateral confinement. These successful results, obtained by this dynamic mesh numerical method, led us to give a solution to this problem for non-Newtonian powerlaw fluids, at constant very low Reynolds number and under the negligible viscoelastic components of the fluid assumption. To corroborate the numerical results, we gave the divergence law of the drag and the distribution of the pressure in the gap asymptotically in the lubrication limit. The successful confrontation of both methods confirms their validity. This new result may find an application in the physics of aggregation of particles in dispersions as well as in the measurement made with the Dynamic Surface Force Apparatus for nanorheology.

\section{Appendix A. Asymptotic results}

In the limit of the lubrication regime $\varepsilon \ll 1$ and at very low Reynolds numbers, when the sphere approaches the wall at constant velocity in unbounded lateral medium (see Fig. 2), the drag force is controlled principally by the drainage process of the liquid film located in the minimum gap remaining between the sphere and the plane. In this situation, the drag force undergone by a sphere can be calculated from the pressure force induced by the radially ejected flow. Note that this drainage taking place in this gap, for the power-law fluid, is reduced to a "radial power-law Poiseuille flow" as shown in Fig. 2 where $a \varepsilon_{1}(\theta)=a \varepsilon+a(1-\cos \theta)$ and $a \varepsilon$ is the minimum gap between the sphere and the plane. Indeed, starting from the following reduced momentum and continuity equations in the lubrication limit:

$\frac{\partial}{\partial z}\left[m\left|\frac{\partial u_{r}(r, z)}{\partial z}\right|^{n}\right]=\frac{\partial p}{\partial r}$

$\frac{1}{r} \frac{\partial}{\partial r}\left[r u_{r}(r, z)\right]+\frac{\partial u_{z}}{\partial z}=0$

By successive integrations and using the boundary conditions, one can obtain:

$u_{r}(r, z)=\frac{n}{n+1}\left[-\frac{1}{m} \frac{\partial p}{\partial r}\right]^{\frac{1}{n}}\left[\left|z-\frac{a \varepsilon_{1}(\theta)}{2}\right|^{1+\frac{1}{n}}-\left(\frac{a \varepsilon_{1}(\theta)}{2}\right)^{1+\frac{1}{n}}\right]$

Then by integrating the continuity Eq. (A.2) over the gap and taking into account that the velocity of the particle $\left.u_{z}(r, z)\right|_{\text {sphere }}=-U$

$\frac{1}{r} \int_{-\frac{a \varepsilon_{1}}{2}}^{+\frac{a \varepsilon_{1}}{2}} \frac{\partial}{\partial r}\left[r u_{r}(r, z)\right] \mathrm{d} z+\int_{-\frac{a \varepsilon_{1}}{2}}^{+\frac{a \varepsilon_{1}}{2}} \frac{\partial u_{z}(r, z)}{\partial z} \mathrm{~d} z=0$

where $u_{r}(r, z)$ is given by Eq. (A.3) and $u_{z}\left(r, z=+a \varepsilon_{1} / 2\right)=-U$ on the sphere and $u_{z}\left(r, z=-a \varepsilon_{1} / 2\right)=0$ on the plane, it is possible to obtain the radial pressure distribution along the gap which is given by:

$p\left(r_{+}\right)-p_{\infty}=-m U^{n}\left(\frac{2 n+1}{n}\right)^{n} \times\left(\frac{2^{\frac{n+1}{2}}}{a^{n} \varepsilon^{\frac{3 n+1}{2}}}\right) \int_{\infty}^{\frac{1}{2 \varepsilon} r^{2}+} \frac{Y^{\frac{n-1}{2}}}{[Y+1]^{2 n+1}} \mathrm{~d} Y$

where $Y=\frac{1}{2} \frac{1}{\varepsilon}\left(\frac{r}{a}\right)^{2}$. Using the MATHEMATICA code, the normalized radial distribution of the pressure is given by:

$$
\begin{aligned}
\frac{p\left(r_{+}\right)-p_{\infty}}{m\left(\frac{U}{2 a}\right)^{n}}= & \left(\frac{2 n+1}{n}\right)^{n}\left(\frac{2^{2+3 n}}{1+3 n}\right)\left(r_{+}\right)^{-(1+3 n)} \\
& \times{ }_{2} F_{1}\left(1+2 n, \frac{1}{2}(1+3 n) ; \frac{3}{2}(1+n) ;-2 \frac{\varepsilon}{r_{+}^{2}}\right)
\end{aligned}
$$

where $p_{\infty}$ is the pressure far from the gap, $r_{+}=r / a$ is the normalized radial distance from the stagnation point and ${ }_{2} F_{1}$ is the Gaussian hypergeometric function. Note that this result is similar to that obtained from Rodin's formula (12) in Section 3. In this approach, the correction factor $\delta(n, k=0, \varepsilon)$ of the drag undergone by a sphere in lateral unbounded medium can be calculated by integrating the pressure given by formula (A.6) over the frontal surface of the sphere in the lubrication limit:

$$
\begin{aligned}
\delta(n, k & =0, \varepsilon)=\frac{F(n, k=0, \varepsilon)}{F(n, k=0, \varepsilon \rightarrow \infty)} \\
& =\frac{2^{\frac{3 n+3}{2}}}{3\left(9 n^{2}-1\right)}\left(\frac{2 n+1}{n}\right)^{n} \times \frac{\Gamma\left(\frac{3 n+3}{2}\right) \Gamma\left(\frac{n+3}{2}\right)}{\Gamma(2 n+1)} \frac{1}{\varepsilon^{\frac{3 n-1}{2}}}
\end{aligned}
$$

This formula, which is valid mathematically only for $n>1 / 3$, reduces to the formula (14) deduced from Rodin's result in the limit of $\beta=\infty$ (Section 3). In fact, to verify the equivalency between both formulae, let us recall that [28]:

$\frac{4}{9 n^{2}-1} \frac{\Gamma\left(\frac{3+n}{2}\right) \Gamma\left(\frac{3 n+3}{2}\right)}{\Gamma(2 n+1)}=\beta\left(\frac{3+n}{2}, \frac{3 n-1}{2}\right)$

\section{References}

[1] P.G. de Gennes, Dynamics of concentrated dispersions: a list of problems, Physico-Chem. Hydrodyn. 2 (1981) 31-44. 
[2] F. Restagno, J. Crassous, E. Charlaix, C. Cottin-Bizonne, M. Monchanin, A new surface apparatus for nanorheology, Rev. Scient. Instrum. 73 (2002) 2292-2297.

[3] C. Cottin-Bizonne, Hydrodynamique à l'interface solide-liquide: étude par mesures de forces de surfaces et simulations de dynamique moléculaire, Ph.D. thesis, Université Claude Bernard Lyon I, 2003.

[4] R. Cox, H. Brenner, The slow motion of a sphere through a viscous fluid towards a plane surface. Part ii. Small gaps widths, including inertial effects, Chem. Eng. Sci. 22 (1967) 1753-1777.

[5] M. Stimson, G.B. Jeffery, The motion of two spheres in a viscous fluid, Proc. R. Soc. Lond. Ser. A 111 (1926) 110-116.

[6] H. Brenner, The slow motion of a sphere through a viscous fluid towards a plane surface, Chem. Eng. Sci. 16 (1961) 242-251.

[7] A.D. Maude, End effects in a falling-sphere viscometer, Br. J. Appl. Phys. 12 (1961) 293-295.

[8] A. Ambari, B. Gauthier-Manuel, E. Guyon, Wall effects on a sphere translating at constant velocity. J. Fluid Mech. 149 (1984) 235-253.

[9] A. Ambari, B. Gauthier-Manuel, E. Guyon, Direct measurement of tube wall effect on the stokes force, Phys. Fluids 28 (1985) 1559-1561.

[10] A. Ben Richou, A. Ambari, J.K. Naciri, Correction factor of the stokes force undergone by a sphere in the axis of a cylinder in uniform and poiseuille flows, Eur. Phys. J. Phys. 24 (2003) 153-165.

[11] G.J. Rodin, Squeeze film between two spheres in a power-law fluid, J. Non-Newton. Fluid Mech. 63 (1996) 141-152.

[12] A.M. Ardekani, R.H. Rangel, D.D. Joseph, Motion of a sphere normal to a wall in a second-order fluid, J. Fluid Mech. 587 (2007) 163-172.

[13] J.D. Sherwood, Squeeze flow of a power-law fluid between non-parallel plates, J. Non-Newton. Fluid Mech. 166 (2011) 289-296.

[14] A.M. Ardekani, D.D. Joseph, D. Dunn-Rankin, R.H. Rangel, Particle-wall collision in a viscoelastic fluid, J. Fluid Mech. 633 (2009) 475-483.

[15] P.J. Carreau, D.C.R.D. Kee, R.P. Chhabra, Rheology of Polymeric Systems: Principles and Applications, Hanser Gardner Publications, 1997.
[16] A. Despeyroux, A. Ambari, A. Ben Richou, The hydrodynamic interaction effects on the settling and the transportation of a sphere in power-law fluids, Phys. Fluids in June 2011, in preparation.

[17] ANSYS, Inc., ANSYS FLUENT Theory Guide (November 2010).

[18] L. Pasol, M. Chaoui, S. Yahiaoui, F. Feuillebois, Analytical solutions for a spherical particle near a wall in axisymmetrical polynomial creeping flows, Phys. Fluids 17 (2005) 073602.

[19] D.Y. Chan, R.G. Horn, The drainage of thin liquid films between solid surfaces, J. Chem. Phys. 83 (1985) 5311-5324.

[20] A. Mongruel, N. Lecoq, E. Wajnryb, B. Cichocki, F. Feuillebois, Motion of a sphero-cylindrical particle in a viscous fluid in confined geometry, Eur. J. Mech. B/Fluids 30 (2011) 405-408.

[21] A. Mongruel, C. Lamriben, S. Yahiaoui, F. Feuillebois, The approach of a sphere to a wall at finite reynolds number, J. Fluid Mech. 661 (2010) 229-238.

[22] G.G. Joseph, R. Zenit, M.L. Hunt, A.M. Rosenwinkel, Particle-wall collisions in a viscous fluid, J. Fluid Mech. 433 (2001) 329-346.

[23] P. Gondret, M. Lance, L. Petit, Bouncing motion of spherical particles in fluids, J. Fluid Mech. 14 (2002) 643-652.

[24] J.O. Marston, W. Yong, S.T. Thoroddsen, Direct verification of the lubrication force on a sphere travelling through a viscous film upon approach to a solid wall, J. Fluid Mech. 655 (2010) 515-526.

[25] P.G. de Gennes, Coil-stretch transition of dilute flexible polymers under ultrahigh velocity gradients, J. Chem. Phys. 60 (1974) 5030-5042.

[26] A. Ambari, C. Deslouis, B. Tribollet, Coil-stretch transition of macromolecules in laminar flow around a small cylinder, Chem. Eng. Commun. 29 (1984) 6378.

[27] E.J. Hinch, Mechanical models of dilute polymer solutions for strong flows with large polymer deformations, in: Colloque Internatioanl CNRS: polymère et lubrification, Brest, France, 1974, pp. 241-247.

[28] I.S. Gradshteyn, I.M. Ryzhik, Tables of integrals, series, and products, 4th ed., Academic Press, Inc., Orlando, 1983. 